\title{
Interacting Frobenius Algebras are Hopf
}

\author{
Ross Duncan Kevin Dunne \\ University of Strathclyde \\ 26 Richmond Street, Glasgow, G1 1XH, UK.
}

\begin{abstract}
Theories featuring the interaction between a Frobenius algebra and a Hopf algebra have recently appeared in several areas in computer science: concurrent programming, control theory, and quantum computing, among others. Bonchi, Sobocinski, and Zanasi [9] have shown that, given a suitable distribution law, a pair of Hopf algebras forms two Frobenius algebras. Here we take the opposite approach, and show that interacting Frobenius algebras form Hopf algebras. We generalise [9] by including non-trivial dynamics of the underlying object - the so-called phase group — and investigate the effects of finite dimensionality of the underlying model, and recover the system of Bonchi et al as a subtheory in the prime power dimensional case. However the more general theory does not arise from a distributive law.
\end{abstract}

Categories and Subject Descriptors F [3]: 2; F [4]: 1; F [4]: 3

\section{Introduction}

Frobenius algebras and bialgebras are structures which combine a monoid and a comonoid on a single underlying object. They have a long history ${ }^{1}$ in group theory, but have applications in many other areas: natural language processing [29, 30], topological quantum field theory [24], game semantics [26], automata theory [36], and distributed computing [7], to name but a few.

In quantum computation, the bialgebraic interplay between two Frobenius algebras describes the behaviour of complementary observables [13, 16], a central concept in quantum theory. This interaction is the basis of the ZX-calculus, a formal language for quantum computation. Using these ideas, a significant fraction of finite dimensional quantum theory can be developed without reference to Hilbert spaces. Surprisingly, almost exactly the same axioms have also appeared in totally different settings: Petri nets $[11,33]$ and control theory [6, 10]. This combination of structures seems to have broad relevance in computer science.

The approach of the current paper is directly inspired by the recent work of Bonchi, Sobociński, and Zanasi [9], who investigated the theory of interacting Hopf algebras ${ }^{2}$ and showed that Hopf

\footnotetext{
${ }^{1}$ See Fauser [21] for much detail on Frobenius algebras, including their history; for the history of Hopf algebras see [2].

${ }^{2}$ A Hopf algebra is a bialgebra with some extra structure; see later 6.2
}

Permission to make digital or hard copies of part or all of this work for personal or classroom use is granted withou fee provided that copies are not made or distributed for profit or commercial advantage and that copies bear this notice and the full citation on the first page. Copyrights for components of this work owned by others than ACM must be honored. Abstracting with credit is permitted. To copy otherwise, to republish, to post on servers, or to redistribute to lists, contact the Owner/Author. Request permissions from permissions@ @ acm.org or Publications Dept., ACM, Inc., fax +1 (212) 869-0481. Copyright 2016 held by Owner/Author. Publication Rights Licensed to ACM.

LICS '16, July 05-08, 2016, New York, NY, USA

DOI: http://dx.doi.org/10.1145/2933575.2934550 algebras which obey a certain distributive law form Frobenius algebras [8, 9]. Using Lack's technique of composing PROPs [25], they show the resulting theory $\mathbb{I H}_{R}$ is isomorphic to that of linear relations 3

Do interacting quantum observables [16] admit such a beautiful description? In this paper we present a rational reconstruction of the theory of strongly complementary observables and show that, except under quite restrictive circumstances, the theory does not arise by composing PROPs via a distributive law. Along the way we also clarify the structure of the theory of complementary observables and show that some assumptions used in earlier work are unnecessary.

In the quantum context, the key insight is that an observable of some quantum system corresponds to a Frobenius algebra on its state space [15]. Further, the state spaces have non-trivial endomorphims giving their internal dynamics; among these there is a phase group for each observable, which leaves the observable unchanged ${ }^{4}$ Since observables are fundamental to quantum theory, we take Frobenius algebras and their phase groups as the starting point, and freely construct $\mathbf{F} G$, the PROP of a Frobenius algebra with a given group of phases $G$.

The general plan of the paper is to begin with a pair of such Frobenius algebras and formalise interactions between them by imposing stronger and stronger axioms upon them. We produce a series of PROPs

$$
\mathrm{F}_{-}+\mathrm{F}_{-} \longrightarrow \mathrm{IF} \longrightarrow \mathrm{IFK} \longrightarrow \mathrm{IFK}_{\mathrm{d}}
$$

each more closely resembling quantum theory than its predecessor. The first is simply the disjoint union of two non-interacting observables. The second requires that the observables be strongly complementary; this means their corresponding Frobenius algebras jointly form a Hopf algebra [13, 16]. The additional structure allows us to construct a ring of endomorphisms of the generator, distinct from the phase groups, and a large class of multiparty unitary operations, being the abstract counterpart of quantum circuits. The next two PROPs introduce eigenstates for the observables, and the effect of finite dimensionality of the state space respectively. In the last of these, $\mathbf{I F K}_{\mathbf{d}}$, if the dimension is a prime power then we recover the system $\mathbb{I} \mathbb{H}_{R}$ of Bonchi et al [9] as a subcategory.

Each of these theories is actually a functor from a suitable category of groups, so we can freely construct a quantum-like theory with any given dynamics. We view the PROPs as syntactic objects, being the graphical language of a theory. However, at several points in the paper, we consider properties of their models (i.e. their algebras), and use these properties as a justification for the next PROP in the chain.

Our motivation for studying these generalisations is to better understand categorical quantum theory [1], particularly with a view

\footnotetext{
${ }^{3}$ Baez and Erbele [6] prove the same result with different techniques.

${ }^{4}$ The importance of the phase group in non-locality arguments has been demonstrated by Edwards [20]
} 
to the ZX-calculus. We explicate the necessary features of higher dimensional versions of the calculus, and separate the algebraic foundation from model-specific details. This will help clarify questions of completeness [3, 5, 19, 27] and also aid in the formalisation of error correcting codes [18]. However given the interest in these structures in other areas, we expect that a richer theory will lead to unexpected applications elsewhere.

Due to restriction on space the proofs are mostly omitted.

\section{Background}

We assume that the reader is familiar with the theory of monoidal categories; all monoidal structures here are taken to be strict. We will employ diagrammatic notation throughout the paper; see Selinger [32]. Our convention is to read diagrams from top to bottom; however, since we operate in a †-category, every diagram can be read equally well from bottom to top; the reader who chooses to do so will need to add the involutive prefix "co" throughout the text themselves.

Definition 2.1. A $\dagger$-category is a category $\mathcal{C}$ equipped with a functor $\dagger: \mathcal{C}^{\text {op }} \rightarrow \mathcal{C}$ which is involutive and acts as the identity on objects.

A morphism $f: A \rightarrow B$ in a $\dagger$-category is called unitary if $f^{\dagger}: B \rightarrow A$ is the two-sided inverse of $f$; it is self-adjoint if $f=f^{\dagger}$.

Remark 2.2. A groupoid is a $\dagger$-category in which every morphism is unitary; in particular every group can be viewed as a one-object $\dagger$-category.

A functor $F: \mathcal{C} \rightarrow \mathcal{D}$ between $\dagger$-categories is a $\dagger$-functor if $(F f)^{\dagger}=F\left(f^{\dagger}\right)$ for all arrows $f$. A (symmetric) monoidal $\dagger$ category is called $\dagger$-(symmetric) monoidal if $-\otimes-: \mathcal{C} \times \mathcal{C} \rightarrow \mathcal{C}$ is a $\dagger$-functor, and all the canonical isomorphisms of the monoidal structure are unitary.

The main example of interest is fdHilb, the category of finite dimensional Hilbert spaces over $\mathbb{C}$ and linear maps; given $f: A \rightarrow$ $B, f^{\dagger}: B \rightarrow A$ is the usual Hermitian adjoint.

We now turn our attention to PROPs. This material largely follows [25] 9].

Definition 2.3. A product category, abbreviated $P R O$, is a strict monoidal category whose objects are generated by a single object under the tensor product; or equivalently, whose objects are the natural numbers. A product and permutation category, abbreviated PROP, is a symmetric PRO. A $\uparrow-P R O$ or $\dagger-P R O P$ is a PRO (respectively PROP) which is also a $\dagger$-monoidal category.

Given any strict monoidal category $\mathcal{C}$ the full subcategory generated by a single object under tensor is a PRO. In particular, for any natural number $D$ we can consider the full subcategory of fdHilb generated by $\mathbb{C}^{D}$ under the tensor product. For $D=2$ this gives the usual setting of quantum computing.

For a PRO $\mathbf{T}$ and a strict monoidal category $\mathcal{C}$, a $\mathbf{T}$-algebra in $\mathcal{C}$ is a strict monoidal functor from $\mathbf{T}$ to $\mathcal{C}$. We will abuse notation and refer to the algebra by the name of its generating object in $\mathcal{C}$. A morphism between PROs is an algebra which is the identity on objects. Therefore we have a category PRO of PROs and their morphisms. The same can be done for PROPs, $\dagger$-PROs, and $\dagger$ PROPs by requiring that the functor is symmetric monoidal and/or dagger as appropriate.

Let $\mathbf{P}$ be the PRO whose morphisms $n \rightarrow n$ are the permutations on $n$ elements, with no morphisms $n \rightarrow m$ if $n \neq m$. $\mathbf{P}$ is groupoid, hence also a $\uparrow$-category. We can understand the category $\dagger$-PROP as a subcategory of the coslice category $\mathbf{P} / \dagger-\mathbf{P R O}$, in which all permutations are natural. The coproduct $\mathbf{T}_{1}+\mathbf{T}_{2}$ in $\dagger$-PROP is given by the pushout of $\mathbf{T}_{1} \leftarrow \mathbf{P} \rightarrow \mathbf{T}_{2}$ in $\dagger$-PRO since the symmetric structure has to agree in both.
In this paper we are concerned with PROPs which are presented syntactically. The arrows of the PROP will be constructed by composition and tensor from the elements of a monoidal signature $\Sigma$ and a set $E$ of equations between terms of the same type. Equality is then the least congruence generated by $E$ and the equations of the symmetric monoidal structure. From this point of view the coproduct $\mathbf{T}_{1}+\mathbf{T}_{2}$ is given by the pair $\left(\Sigma_{1}+\Sigma_{2}, E_{1}+E_{2}\right)$.

The coproduct is not an especially exciting operation: we need to combine PROPs and make them interact. Lack's method of composing PROPs via distributive laws is a particularly elegant approach [25]. We will skip the details here, but given two PROPs $\mathbf{T}_{1}$ and $\mathbf{T}_{2}$, a distributive law $\lambda: \mathbf{T}_{2} ; \mathbf{T}_{1} \rightarrow \mathbf{T}_{1} ; \mathbf{T}_{2}$ is a set of directed equations $\left(f_{2}, f_{1}\right) \rightarrow\left(f_{1}^{\prime}, f_{2}^{\prime}\right)$ commuting morphisms of $\mathbf{T}_{2}$ past those of $\mathbf{T}_{1}$. The composite PROP $\mathbf{T}_{1} ; \mathbf{T}_{2}$ has morphisms of the form $n \stackrel{f_{1}}{\longrightarrow} z \stackrel{f_{2}}{\longrightarrow} m$ where $f_{1}$ is an arrow of $\mathbf{T}_{1}$ and $f_{2}$ of $\mathbf{T}_{2}$; its syntactic presentation is that of $\mathbf{T}_{1}+\mathbf{T}_{2}$ with the additional equations of $\lambda$.

Example 2.4. As a simple example we can view $\mathbf{P}$ as a PRO with a single generator $c: 2 \rightarrow 2$ quotiented by $c^{2}=$ id and the usual hexagon diagrams. Let $G$ be a group; we define $G^{\times}$to be the PRO with hom-sets $G^{\times}(n, n)=\prod_{n} G$, and $G^{\times}(n, m)=\emptyset$ if $n \neq m$. Composition is done component-wise in $G$. The generators of $G^{\times}$ are just the elements $g: 1 \rightarrow 1$ for each $g \in G$ quotiented by the equations of $G$. We can define the composite $\mathbf{P} ; G^{\times}$via the distributive law:

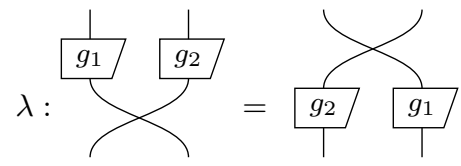

for each $g_{1}$ and $g_{2}$ in $G$. This yields the PRO - actually a $\dagger$-PROP - whose morphisms $n \rightarrow n$ are a permutation on $n$ followed by an $n$-vector of elements of $G$. It's easy to see that this construction yields a functor $\mathbf{P}: \mathbf{G r p} \rightarrow \dagger$-PROP. Notice that $\mathbf{P} G$ is again a groupoid, and every morphism is unitary.

Example 2.5. A second cluster of examples, stolen shamelessly from [25], provides the main structures of interest of this paper. Let $\mathbf{M}$ denote the PROP of commutative monoids; it has two generators, $\mu: 2 \rightarrow 1$ and $\eta: 0: \rightarrow 1$, which we write graphically as $\mathcal{Y}$ and $\mathrm{O}$, subject to the equations:
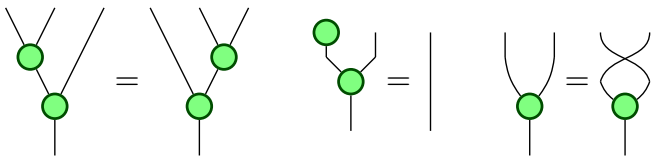

We can define the PROP of cocommutative comonoids as $\mathbf{C}=\mathbf{M}^{\mathrm{op}}$. The generators are $\delta: 1 \rightarrow 2$ and $\epsilon: 1 \rightarrow 0$; the equations are those of $(\mathrm{M}$ ) but flipped upside down. We call these equations (C). Bialgebras and Frobenius algebras combine a monoid and comonoid in different ways; both can be built using distributive laws between $\mathbf{M}$ and $\mathbf{C}$.

Example 2.6. The PROP $\mathbf{B}$ of commutative bialgebras is constructed via a distributive law $\lambda_{B}: \mathbf{M} ; \mathbf{C} \rightarrow \mathbf{C} ; \mathbf{M}$ generated by the equations

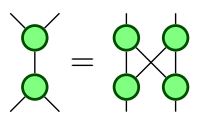<smiles>O=COCCO</smiles><smiles>COOC1OCCO1</smiles> 
where the dashed box represents the empty diagram.

Example 2.7. The PROP $\mathbf{F}$ of Frobenius algebras is also defined by distributive law, $\lambda_{F}: \mathbf{C} ; \mathbf{M} \rightarrow \mathbf{M} ; \mathbf{C}$, given by the equations:
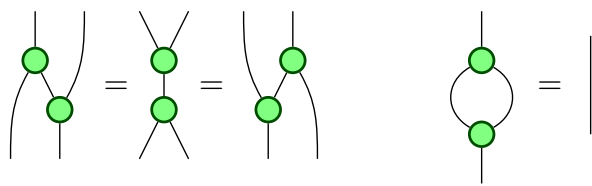

This is not the most general form of Frobenius algebra. More accurately, $\mathbf{F}$ is the PROP of special commutative Frobenius algebras; the last equation above is what makes them "special". Throughout this paper the reader should understand the term "Frobenius algebra" to mean "special commutative $\dagger$-Frobenius algebra", usually abbreviated $\dagger$-SCFA. Rosebrugh, Sabadini, and Walters call the same structure a separable commutative algebra [28].

By defining the PROP F in Example 2.7 via the distributive law $\lambda_{F}$ we can see the following "Spider Theorem" [14], which establishes a normal form for morphisms in the PROP F. In particular every morphism in $\mathbf{F}$ can be expressed as the composition of a morphism in $\mathbf{M}$ followed by a term in $\mathbf{C}$

Theorem 2.8 (Spider Theorem). Let $f: m \rightarrow n$ be a morphism in $\mathbf{F}$; if the graphical form of $f$ is connected then $f=\delta_{n} \circ \mu_{m}$ where

$$
\delta_{0}:=\epsilon \quad \delta_{k+1}:=\left(\delta_{k} \otimes \operatorname{id}_{A}\right) \circ \delta
$$

and $\mu_{m}$ is defined dually.

With this in mind we define a "spider" $\bigcirc_{n}^{m}:=\delta_{n} \circ \mu_{m}$ as a tree of $m$ multiplies followed by a co-tree of $n$ comultiplies. We can view $\mathbf{F}$ as the category of spiders, where composition means fusing connected spiders and removing any self-loops.

Remark 2.9. We note that all of these PROPs also have "semantic" presentations: $\mathbf{M}$ to equivalent to FinSet, the skeletal category of finite sets and functions, while $\mathbf{B}$ and $\mathbf{F}$ are equivalent to $\operatorname{Span}($ FinSet) and Cospan(FinSet) respectively. See [28] and [25]. The spider theorem is equivalent to this last fact.

For any category $\mathbf{T}$, one can view $\mathbf{T}+\mathbf{T}^{\mathrm{op}}$ as a $\dagger$-category, hence in all of the above we may assume that $\delta=\mu^{\dagger}$ and $\epsilon=\eta^{\dagger}$. However it is not always desirable to do so. Whether we view $\mathbf{C} ; \mathbf{M}$ as a PROP or a †-PROP makes a difference when considering its algebras in some other $\dagger$-category. In the sequel we will ignore the $\dagger$-structure of $\mathbf{B}$ but will take $\mathbf{F}$ as the $\dagger$-PROP of $\dagger$-Frobenius algebras. In particulat this means that a Frobenius algebra may be specified by giving either its monoid part $(\mu, \eta)$ or its comonoid part $(\delta, \epsilon)$.

\section{The Standard Model}

The combination of Frobenius and Hopf algebras arises naturally in the study of quantum observables. In this section we present a class of concrete examples that exist in every finite dimensional complex Hilbert space. The starting point is this theorem of Coecke, Pavlovic, and Vicary [15]:

Theorem 3.1. In fdHilb, $\left(\delta_{\circ}, \epsilon_{\circ}\right)$ is a $\dagger-S C F A$ on $A$ iff

$$
\delta_{\circ}:\left|a_{i}\right\rangle \mapsto\left|a_{i}\right\rangle \otimes\left|a_{i}\right\rangle \quad \epsilon_{\circ}: 1 \mapsto\left|a_{i}\right\rangle .
$$

for some orthonormal basis $\left\{\left|a_{i}\right\rangle\right\}_{i}$ of $A$.

For any coalgebra the elements copied by $\delta-$ the $\left|a_{i}\right\rangle$ in the theorem above - are called set-like. So given an orthonormal basis $|0\rangle, \ldots,|D-1\rangle$ for the Hilbert space $\mathbb{C}^{D}$ we get a $\dagger$-SCFA defined as above, whose set-like elements are $|n\rangle$. We can construct another Frobenius algebra by viewing this basis as the elements of the additive group $\mathbb{Z}_{D}$ and forming the group algebra:

$$
\mu_{\bullet}:|n\rangle \otimes|m\rangle \mapsto|n+m\rangle \quad \eta_{\bullet}:|0\rangle \mapsto 1
$$

This is again a $\dagger$-Frobenius algebra, although it is quasi-special [22] rather than special: we have $\mu \circ \delta=D \cdot$ id rather than the usual "special" equation.

This pair of Frobenius algebras are pair-wise Hopf algebras (see Def.6.2 in the sense that $\left(\mu_{\bullet}, \delta_{\odot}\right)$ is a Hopf algebra, as is $\left(\mu_{\odot}, \delta_{\bullet}\right)$.

Remark 3.2. Any finite abelian group $G$ determines such a tuple $\left(\mu_{\circ}, \delta_{\circ}, \mu_{\bullet}, \delta_{\bullet}\right)$, see Table 1 which we will denote $\mathbb{C} G$ and call the group algebra of $G$.

Such pairs of quantum observables are called strongly complementary [17] and are closely related to the Fourier transform [22].

Recall that the dual group $G^{\wedge}$ of a finite abelian group $G$ is the set of group homomorphisms from $G$ into the circle group of unit complex numbers, with multiplication in $G^{\wedge}$ computed point-wise. We have $G \cong G^{\wedge}$, although this isomorphism is not natural. The set-like elements of $\delta \bullet$ are in 1-1 correspondence with elements of the group $G^{\wedge}$, in particular, for a group character $\chi$,

$$
|\chi\rangle:=\sum_{g \in G} \chi(g)|g\rangle
$$

is set-like for $\delta_{\bullet}$. Distinct $|\chi\rangle,\left|\chi^{\prime}\right\rangle$ are orthogonal, so by rescaling we obtain an orthonormal basis, and via Theorem 3.1 a $\dagger$-SCFA as required. Moreover, in fdHilb every pair of interacting $\dagger$-SCFAs is of the form $\mathbb{C} G$ for a finite abelian group $G$ [17].

Aside from providing some intuition for what a pair of interacting Frobenius algebras might be, we will use these examples as a source of counter-models to show that certain equations do not hold in the syntactic PROPs we define in the main body of the paper. Most of this holds for group algebras over arbitrary fields.

\section{Frobenius Algebras and Phases}

By Theorem 3.1 every Frobenius algebra in fdHilb corresponds to a non-degenerate quantum observable: the set-like elements of the coalgebra are the eigenstates of the observable. In this concrete setting, the maps which fix a given observable are of great interest; we call them phases. Before developing this idea in the abstract setting we will recall some properties of $\mathbf{F}$-algebras.

Let $A$ be an $\mathbf{F}$-algebra in some category $\mathcal{C}$; we let $\delta, \mu$ etc stand for their images in $\mathcal{C}$. The following proposition follows from the Spider Theorem.

Proposition 4.1. The PROP $\mathbf{F}$ is $\dagger$-compact [23], with all objects self-dual.

$$
\begin{array}{r}
\text { Proof. Let } d=\mathrm{O}_{2}^{0}=9 \text { and } e=d^{\dagger} \text {. Then } \\
\qquad 9=1=1
\end{array}
$$

by the spider theorem, which makes 1 self-dual; the required cup and cap for the other objects can be easily constructed (although see [31] for the coherence conditions) to make all of $\mathbf{F}$ compact. For $\dagger$-compactness, we require

$$
(\rho)^{\dagger}=\varnothing
$$

which again follows from the spider theorem.

Obviously, compactness of $\mathbf{F}$ implies that any $\mathbf{F}$-algebra is also compact, in particular the inclusion of $\mathbf{F}$ into another PROP. Given a map $f: A \rightarrow A$, we can construct its " $\bigcirc$-transpose", by conjugating with $d$ and $e$ :

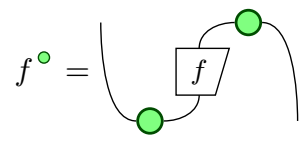


Table 1. Complex Group Algebra ( $e$ the group identity)

\begin{tabular}{|c|c|c|}
\hline & Hopf & Hopf \\
\hline Frobenius & $\begin{array}{c}\mu_{\bullet}::|n\rangle \otimes|m\rangle \mapsto|n+m\rangle \\
\eta_{\bullet}=|e\rangle\end{array}$ & $\delta_{\bullet}::|n\rangle \mapsto \sum_{\substack{m+m^{\prime}=n \\
\epsilon_{\bullet}=\langle e|}}|m\rangle \otimes\left|m^{\prime}\right\rangle$ \\
\hline Frobenius & $\begin{array}{c}\delta_{\circ}::|n\rangle \mapsto|n\rangle \otimes|n\rangle \\
\epsilon_{\circ}=\sum_{n \in G}\langle n|\end{array}$ & $\begin{array}{c}\mu_{\circ}::|n\rangle \otimes|m\rangle \mapsto|n\rangle \text { if } g=h, 0 \text { otherwise } \\
\eta_{\circ}=\sum_{n \in G}|n\rangle\end{array}$ \\
\hline
\end{tabular}

The $\bigcirc$-transpose extends to an involutive contravariant functor on any $\mathbf{F}$-algebra $A$, and since $\mathbf{F}$ is $\dagger$-compact, the adjoint and the $\bigcirc$ -transpose commute, and hence we can define a covariant involution, the O-conjugate:

$$
f_{\circ}=\left(f^{\dagger}\right)^{\circ}=\left(f^{\circ}\right)^{\dagger} .
$$

We say that $f$ is $\bigcirc$-real if $f=f_{\circ}$, or equivalently if $f^{\dagger}=f^{\circ}$. Evidently, the defining maps of the Frobenius algebra are $\bigcirc$-real, as is the symmetry of the monoidal structure, hence in $\mathbf{F}$ itself $f^{\dagger}=f^{\circ}$ for all $f$. This is not true for $\mathbf{F}$-algebras in general.

Before moving on we state a useful lemma.

Lemma 4.2. If a morphism $f$ commutes with both the monoid and comonoid parts of a Frobenius algebra, then it is invertible and $f^{-1}=f^{\circ}$.

We are now ready to develop the abstract theory of phases.

Definition 4.3. A pre-phase for the $\dagger$-SCFA $(A, \delta, \mu)$ is a map $\alpha: A \rightarrow A$ which acts as a strength for the multiplication:

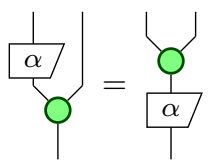

A pre-phase is a phase if it is unitary.

Definition 4.4. Let $\psi: I \rightarrow A$ and define $\Lambda(\psi): A \rightarrow A$ by

$$
\Lambda(\psi): \psi \mapsto \mu \circ(\psi \otimes \mathrm{id})
$$

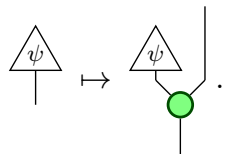

It follows immediately from this definition that $\Lambda(\psi)$ is a prephase. If $\Lambda(\psi)$ is in fact a phase, then we say that $\psi$ is $\bigcirc$-unbiased.

Lemma 4.5. Let $\alpha: A \rightarrow A$ be a phase. Then there exists $\psi: I \rightarrow$ A such that

1. $\alpha=\Lambda(\psi)$;

2. $\alpha^{\circ}=\alpha$;

3. $\alpha^{\dagger}=\Lambda\left(\psi_{\circ}\right)$;

4. $\mu\left(\psi \otimes \psi_{\circ}\right)=\eta$.

Proof. Let $\psi=\alpha \circ \eta$; the rest follows by elementary diagram manipulations.

Corollary 4.6. If $\alpha$ is a phase, then so is $\alpha^{\dagger}$.

Lemma 4.7. Let $\Phi$ denote the set of phases, and $\mathcal{U}$ denote the unbiased points; then $\left(\Phi, \circ, \mathrm{id},()^{\dagger}\right)$ and $\left(\mathcal{U}, \mu, \eta,()_{\circ}\right)$ are isomorphic abelian groups.

We will now consider the $\dagger$-PROP which is generated by a $\uparrow$ SCFA with a prescribed group of phases i.e. where $\left(\Phi, \circ, \mathrm{id},()^{\dagger}\right) \cong$ $G$ for some abelian group $G$. As in example 2.4, given the abelian group $G$ we can construct the PROP $\mathbf{P} G$. We might then hope to compose the PROPs $\mathbf{F}$ and $\mathbf{P} G$ using a distributive law [25], but this is impossible. However, we can form the desired PROP via an iterated distributive law [12] To combine $\mathbf{F}$ and $\mathbf{P} G$ we compose the PROPs $\mathbf{M}, \mathbf{C}$ and $\mathbf{P} G$ pairwise via distributive laws; these distributive laws interact to yield the desired PROP.

Lemma 4.8. 1. The PROPS $\mathbf{M}$ and $\mathbf{P} G$ can be composed via a distributive law $\sigma: \mathbf{P} G ; \mathbf{M} \rightarrow \mathbf{M} ; \mathbf{P} G$, yielding a $P R O P$ presented by the equations of $\mathbf{M}+\mathbf{P} G$ and equation (P1);

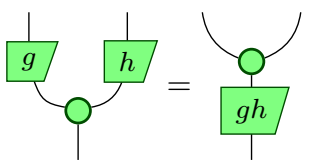

2. The PROPs $\mathbf{C}$ and $\mathbf{P} G$ can be composed via a distributive law $\rho: \mathbf{C} ; \mathbf{P} G \rightarrow \mathbf{P} G ; \mathbf{C}$, yielding a PROP presented by the equations of $\mathbf{C}+\mathbf{P} G$ and equation $(\mathrm{P} 2)$.

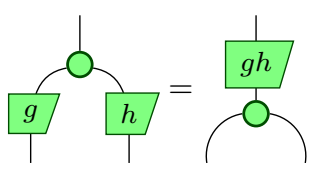

Recall that the PROP $\mathbf{F}$ is defined by a distributed law $\lambda_{F}$ : $\mathbf{C} ; \mathbf{M} \rightarrow \mathbf{M} ; \mathbf{C}$ (Example 2.7).

Theorem 4.9. The distributive laws $\lambda_{F}, \rho$ and $\sigma$ form a distributive series of monads [12], and hence determine a PROP $\mathbf{F} G$ presented by the equations of $\mathbf{M}+\mathbf{P} G+\mathbf{C}$ and equations $[\mathrm{P} 1,[\mathrm{P} 2)$ and $(\mathrm{F})$.

Note that every $\mathbf{F}$-algebra has a group of phases, although it may be the trivial group. We now construct the PROP of Frobenius algebras with a given phase group $G$. Take any abelian group $G$ and consider the †-PROP P $G$ as earlier; then the distributive laws $\rho, \sigma$ and $\lambda_{F}$ allow us to define the functor.

$$
\mathbf{F}: \mathbf{A b} \rightarrow \dagger \text {-PROP . }
$$

For example $\mathbf{F} 1$ is the original PROP of Frobenius algebras $\mathbf{F}$.

The PROPs $\mathbf{F}$ and $\mathbf{P} G$ embed in $\mathbf{F} G$, and equation (P1) ensures that the morphisms $1 \rightarrow 1$ in $\mathbf{P} G$ are phases for the $\dagger$-SCFA i.e. that they satisfy equation $(\boldsymbol{\Phi})$.

Corollary 4.10. Let $f: n \rightarrow n^{\prime}$ in $\mathbf{F} G$; then

$$
f=n \stackrel{\nabla}{\longrightarrow} m \stackrel{g}{\longrightarrow} m \stackrel{\Delta}{\longrightarrow} n^{\prime}
$$

where $\nabla: n \rightarrow m$ is in $\mathbf{M}, \Delta: m \rightarrow n^{\prime}$ is in $\mathbf{C}, g: m \rightarrow m$ is in $G^{\times}$and $m \leq n, n^{\prime}$.

Note that $\mathbf{F} G$-algebras (i.e. models of $\mathbf{F} G$ ) may have many more phases than those from $G$. For a given $\mathbf{F} G$ algebra we will denote the full group of phases $\Phi$, of which $G$ is necessarily a subgroup. Just as $\mathbf{F} G$ generalises $\mathbf{F}$, Corollary 4.10 lets us generalise the Spider Theorem.

Theorem 4.11 (Generalised Spider). Let $f: A^{\otimes m} \rightarrow A^{\otimes n}$ be a morphism built from $\delta, \epsilon, \mu, \eta$, and some collection of phases $\alpha_{i} \in \Phi$ by composition and tensor; if the graphical form of $g$ is connected 
then $f=\delta_{n} \circ \alpha \circ \mu_{m}$ where

$$
\alpha=\alpha_{1} \circ \cdots \circ \alpha_{k}
$$

Therefore a Frobenius algebra and its group of phases generate a category of $\Phi$-labelled spiders. Composition is given by fusing connected spiders and summing their labels.

In particular, if $n=n^{\prime}=1$ in the above then $f$ is either a phase map or a "projector" $\phi \circ \psi^{\dagger}$ for a pair of unbiased points $\phi, \psi: 0 \rightarrow 1$. The following is a consequence of Theorem 4.11

Lemma 4.12. Suppose $f: n \rightarrow n$ is unitary in $\mathbf{F} G$; then $f \in \mathbf{P} G$.

\section{Two Frobenius Algebras}

We briefly consider the structure of the free $\dagger$-PROP $\mathbf{F} G+\mathbf{F} H$, i.e. the case of two non-interacting Frobenius algebras.

Notation We will adopt the convention that elements in image of the first injection (i.e. from $\mathbf{F} G$ ) are coloured green and the elements in the second $(\mathbf{F} H)$ are coloured red. In practice, the colour we call "green" may be light grey, and "red" may be dark grey depending how you read this document.

Morphisms of $\mathbf{F} G+\mathbf{F} H$ are alternating sequences of morphisms from $\mathbf{F} G$ and $\mathbf{F} H$; i.e. $f=g_{1} \circ h_{1} \circ g_{2} \circ h_{2} \circ \cdots \circ g_{n} \circ h_{n}$ where $g_{i} \in \mathbf{F} G$ and $h_{j} \in \mathbf{F} H$. Although no equations force the two components to interact, the spider theorem holds separately in each colour, hence any morphism can be reduced to a 2-coloured graph, and any 2-coloured (self-loop free) graph is valid morphism. The following is a consequence of Lemma 4.12

Lemma 5.1. Let $u: n \rightarrow n$ be unitary in $\mathbf{F} G+\mathbf{F} H$; then $u$ is in $\mathbf{P} G+\mathbf{P} H$.

As a special case of the above, if $u: 1 \rightarrow 1$ is unitary, it is an element of the free product of groups $G * H$. However, unlike in F $G$ this group structure is not reflected back to the points, since we have to choose between $\mu_{\circ}$ and $\mu_{\bullet}$ for the multiplication, and the wrong colour merely generates the free monoid on $G$ rather than reproducing the group structure.

In $\mathbf{F} G+\mathbf{F} H$ we have two distinct transposition and conjugation operations which do not coincide, i.e. $f^{\circ} \neq f^{\bullet}$.

Lemma 5.2. Let $f: n \rightarrow n$ be a morphism in $\boldsymbol{F} 1+\boldsymbol{F} 1$; then $f$ is O-real iff it is green and 0 -real iff it is red.

Corollary 5.3. In $\boldsymbol{F} 1+\boldsymbol{F} 1, f_{\circ}=f_{\bullet}$ implies $f \in \boldsymbol{P} 1$.

\section{Interacting Frobenius Algebras}

The notion of two observables being complementary is central to the theory of quantum mechanics. In categorical quantum mechanics strong complementarity is characterised by a pair of Frobenius algebras jointly forming a Hopf algebra [16].

We now impose some equations on $\mathbf{F} G+\mathbf{F} H$ governing their interaction. We want $\mathbf{F} G$ and $\mathbf{F} H$ to jointly form a bialgebra so we impose:

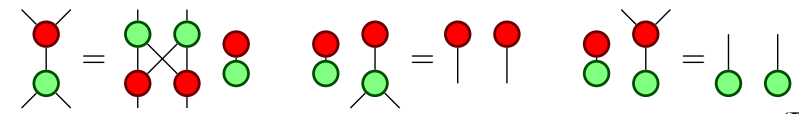

We call the resulting structure a Frobenius bialgebra: the pairs $\left(\delta_{\circ}, \mu_{\circ}\right)$ and $\left(\delta_{\bullet}, \mu_{\bullet}\right) \dagger$-SCFAs, while the pairs $\left(\delta_{\circ}, \mu_{\bullet}\right)$ and $\left(\delta_{\bullet}, \mu_{\circ}\right)$ are bialgebras.

Remark 6.1. This definition differs from the usual one by the presence of the scalar factor $\epsilon_{\circ} \eta_{\bullet}$ in the equations, and the omission of the equation:

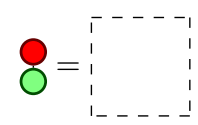

In [16] this structure is called a scaled bialgebra. The usual definition can be restored by imposing $(\vec{B})$. Space does not permit a full discussion of the scalars but note that equation $\left(\overline{B^{3}}\right)$ is not true in the standard model $\mathbb{C Z}_{D}$. However, having belaboured the point that the scalars are needed, we henceforward omit them in the name of clarity - they can always be restored if needed: see Backens [4].

Definition 6.2. A bialgebra on $A$ is called a Hopf algebra if there exists $s: A \rightarrow A$, called the antipode, satisfying the equation

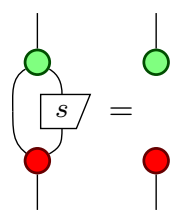

Definition 6.3. Let $\left(\delta_{\circ}, \epsilon_{\circ}, \mu_{\bullet}, \eta_{\bullet}\right)$ be a Frobenius bialgebra as above; define the antipode $s$ as

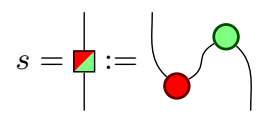

Theorem 6.4. The morphisms $\left(\delta_{\circ}, \epsilon_{\circ}, \mu_{\bullet}, \eta_{\bullet}\right)$ form a Hopf algebra if and only if $\eta_{\bullet}=\left(\epsilon_{\bullet}\right)^{\circ}$ and $\epsilon_{0}=\left(\eta_{\circ}\right)^{\bullet}$, i.e.

$$
\mathcal{Q}=0 \quad \delta=9
$$

Remark 6.5. In the original paper on interacting quantum observables [16] the condition "O-classical points are $\bigcirc$-real" formed part of the definition of complementarity; equation + is a weakening of this condition.

Equation $\square$ can be stated in purely Hopf algebraic terms as

$$
\eta=0 \quad \eta=0,
$$

however the given version emphasises that it is an interaction of the red and green monoid structures, but not a complete distributive law. Indeed, as we shall see later, there is no general distributive law of $\mathbf{F} G$ over $\mathbf{F} H$. We are forced to define the PROP of interacting Frobenius algebras as a quotient.

Definition 6.6. Let $\mathbf{I F}(G, H)$ be the PROP obtained quotienting $\mathbf{F} G+\mathbf{F} H$ by the equations $(\mathrm{B}+)$. This gives a functor $\mathbf{I F}: \mathbf{A b} \times$ Ab $\rightarrow$ †-PROP.

Whenever the groups $G$ and $H$ are obvious or unimportant, we abbreviate $\mathbf{I F}(G, H)$ by IF.

Example 6.7. The group algebras $\mathbb{C Z}_{D}$ described in Section 3 are $\mathbf{I F}\left(\mathbb{Z}_{D}, \mathbb{Z}_{D}\right)$-algebras. Indeed, the same group algebras are models of $\mathbf{I F}\left(T^{D-1}, T^{D-1}\right)$, where $T^{n}$ is the $n$-torus, i.e. the $n$ fold product of circles. For $D=2$ this yields the usual model of the ZX-calculus.

IF contains two copies of the PROP of bialgebras: $\mathbf{B}$ generated by $\left(\delta_{\circ}, \mu_{\bullet}\right)$ and $\mathbf{B}^{\text {op }}$ by $\left(\delta_{\bullet}, \mu_{\odot}\right)$. By Theorem 6.4 IF also contains two Hopf algebra structures. Let $\mathbf{H A}$ be the subcategory generated by $\mathbf{B}$ and $s$, and define $\mathbf{H A}^{\mathrm{op}}$ dually. Note that we have an isomorphism $\mathbf{H A} \cong \mathbf{H A}^{\text {op }}$ via the dagger.

Proposition 6.8. Let $s$ be the antipode of a commutative Hopf algebra $H$; then

1. $s$ is the unique map satisfying $(\mathrm{H})$;

2. $s$ is a bialgebra morphism;

3. $s \circ s=\mathrm{id}$; 
4. Let $K$ be a commutative Hopf algebra with antipode $s^{\prime}$; then for any bialgebra morphism $f: H \rightarrow K$ we have $f \circ s=s^{\prime} \circ f$

These are standard properties (see [34]) which lead immediately to the following.

Corollary 6.9. Let $s$ be as defined in 6.3. then:

1. $s$ is a self-adjoint unitary

2. $s=(s)_{\circ}=(s) \bullet$

Remark 6.10. Since the Hopf algebra is built out of $\dagger$-Frobenius algebras, and $s=s^{\dagger}$, we know that $s$ is also the antipode for the opposite bialgebra.

Corollary 6.9 implies that the antipode commutes with all of the structure in of $\mathbf{I F}(1,1)$. This forces the two transpositions to interact in a variety of unexpected ways.

Lemma 6.11. For any $f: n \rightarrow m$ we have $f^{\circ}=f^{\bullet}$.

Proof.

$$
f^{\circ \bullet}=\left(s^{\otimes m}\right) \circ f \circ\left(s^{\dagger \otimes n}\right)=\left(s^{\dagger \otimes m}\right) \circ f \circ\left(s^{\otimes n}\right)=f^{\bullet \circ} .
$$

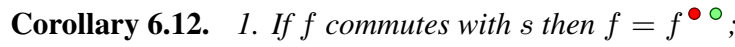

2. If $f$ commutes with $s$ then $f$ is $\mathrm{O}$-real iff it is $\mathrm{O}$-real;

3. If $f$ is both $\mathrm{O}$-real and $\mathrm{O}$-real then it commutes with $\mathrm{s}$.

Proof. 1.

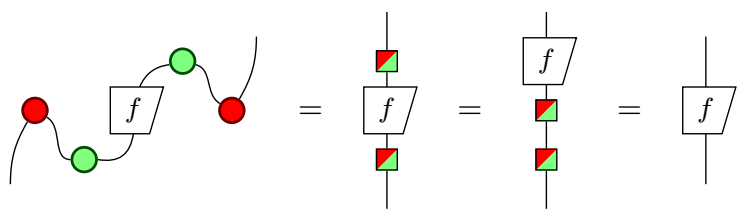

2. Suppose $f^{\dagger}=f^{\circ}$, then by the above $f=f^{\circ \bullet}=f^{\dagger}=f_{\bullet}$ The converse holds by the same argument.

3. Suppose $f$ is $\bigcirc$-real and $\bigcirc$-real; then $f=f^{\bullet \circ}=s f s$ which gives the result by Corollary 6.83 .

Corollary 6.13. Suppose $k: 0 \rightarrow 1$ is $\mathrm{O}$-real, and let $h=\Lambda_{\bullet}(k)$. Then

1. $s \circ k=k_{\bullet}$, and

2. $s \circ h \circ s=h^{\dagger}$.

Proposition 6.14. In $\mathbf{I F}(1,1) f^{\circ}=f^{\bullet}=f^{\dagger}$ for all morphisms $f$.

Proof. The generators of $\mathbf{I F}(1,1)$ are real in their own colour, and as noted above $s$ commutes with all the generators of the PROP; hence the result follows by Corollary 6.12

Given a pair of bialgebras $\left(A, \mu_{A}, \delta_{A}\right)$ and $\left(B, \mu_{B}, \delta_{B}\right)$, the collection of morphisms $A \rightarrow B$ becomes a monoid under the convolution product where $f+g:=\mu_{B} \circ(f \otimes g) \circ \delta_{A}$, and the unit is $\eta_{A} \circ \epsilon_{B}$.

In particular the endomorphisms of the bialgebra $\left(\delta_{\circ}, \epsilon_{\odot}, \mu_{\bullet}, \eta_{\bullet}\right)$ carry this monoid structure.

Proposition 6.15. Let $f$ be a bialgebra morphism, and $s$ the antipode; then for all $g, h: A \rightarrow A$ we have:

1. $f \circ(g+h)=(f \circ g)+(f \circ h)$,

2. $(g+h) \circ f=(g \circ f)+(h \circ f)$, and

3. $f+(f \circ s)=0$.

Lemma 6.16. If $f$ and $g$ are bialgebra morphisms then so is $f+g$.
Hence the bialgebra morphisms of $\left(\delta_{\circ}, \epsilon_{\circ}, \mu_{\bullet}, \eta_{\bullet}\right)$ form a unital ring $R$, with multiplication given by composition, and where the additive inverse is given by composing with $s$.

Define $\mathbf{n}: A \rightarrow A$ by

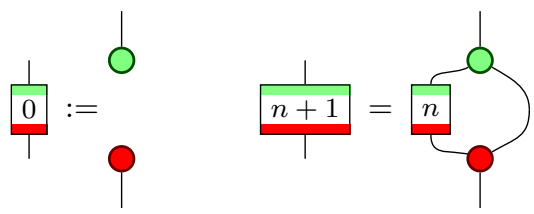

for all $n \in \mathbb{N}$. Accordingly we refer to the morphisms $\mathbf{n} \in \mathbb{Z}$ as the internal integers. Applying the bialgebra law and the spider theorem respectively we have:

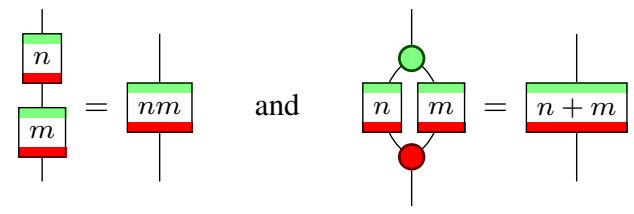

Further, $\mathbf{n}$ is a bialgebra morphism for $\left(\delta_{\circ}, \epsilon_{\circ}, \mu_{\bullet}, \eta_{\bullet}\right)$.

Example 6.17. In the group algebra $\mathbb{C Z}_{3}$ the internal integers are given by the following matrices:

$$
\mathbf{0}=\left(\begin{array}{lll}
1 & 0 & 0 \\
1 & 0 & 0 \\
1 & 0 & 0
\end{array}\right) \quad \mathbf{1}=\left(\begin{array}{lll}
1 & 0 & 0 \\
0 & 1 & 0 \\
0 & 0 & 1
\end{array}\right) \quad \mathbf{2}=\left(\begin{array}{lll}
1 & 0 & 0 \\
0 & 0 & 1 \\
0 & 1 & 0
\end{array}\right)
$$

There are no others; see Section 8 for discussion.

Lemma 6.18. Let $\alpha$ be a phase of either colour in $\mathbf{I F}(G, H)$; then $\alpha$ is bialgebra morphism iff $\alpha=\mathrm{id}$.

Since the non-trivial phases can never be bialgebra morphisms, we restrict our attention to $\mathbf{I F}(1,1)$ for the rest of this section.

In any PROP, given a monoid $(\mu, \eta)$ on 1 , a monoid on 2 can be defined using the tensor:

$$
\mu_{2}:=\text { O } \eta_{2}:=99
$$

and similarly for a comonoid $(\delta, \epsilon)$. It is easy to check that if $(\delta, \epsilon, \mu, \eta)$ is a bi-, Hopf, or Frobenius algebra then so is $\left(\delta_{2}, \epsilon_{2}, \mu_{2}, \eta_{2}\right)$. Continuing in the same way, there is a Hopf algebra on every object $n$ of HA. Therefore all the preceding discussion applies equally well to bialgebra morphisms $n \rightarrow m$ for any $n$ and $m$. In particular all the generators of $\mathbf{H A}$ are bialgebra morphisms, which yields:

Lemma 6.19. Every morphism in HA is a bialgebra homomorphism for $\left(\delta_{\circ}, \mu_{\bullet}\right)$.

Thanks to $\dagger$-duality, any bialgebra morphism for $f \in \mathbf{H A}$ gives a bialgebra morphism $f^{\dagger} \in \mathbf{H A}^{\mathrm{op}}$, so we also have an isomorphic opposite ring $R^{\text {op }}$, complete with opposite integers $\mathbf{n}^{\dagger}$. None of the equations of IF forces these structures to interact: there is no commutation of $\delta_{\circ}$ and $\delta_{\bullet}$ for example. However, if we restrict attention to the invertible morphisms of $\mathbf{I F}(1,1)$ we can make some progress.

Lemma 6.20. If $f$ is an invertible bialgebra morphism then $f^{-1}$ is also a bialgebra morphism.

Lemma 6.21. Let $f: n \rightarrow n$ be a bialgebra morphism, and suppose that $f^{\prime} \circ f=f \circ f^{\prime}=\mathrm{id}$ for some morphism $f^{\prime}: n \rightarrow n$, which is both O-real and O-real; then $f^{\prime}=f^{\dagger}$.

Proof. By uniqueness of inverses in monoids it is enough to show that $\left(-f^{\prime}\right)+f^{\dagger}=0$. 

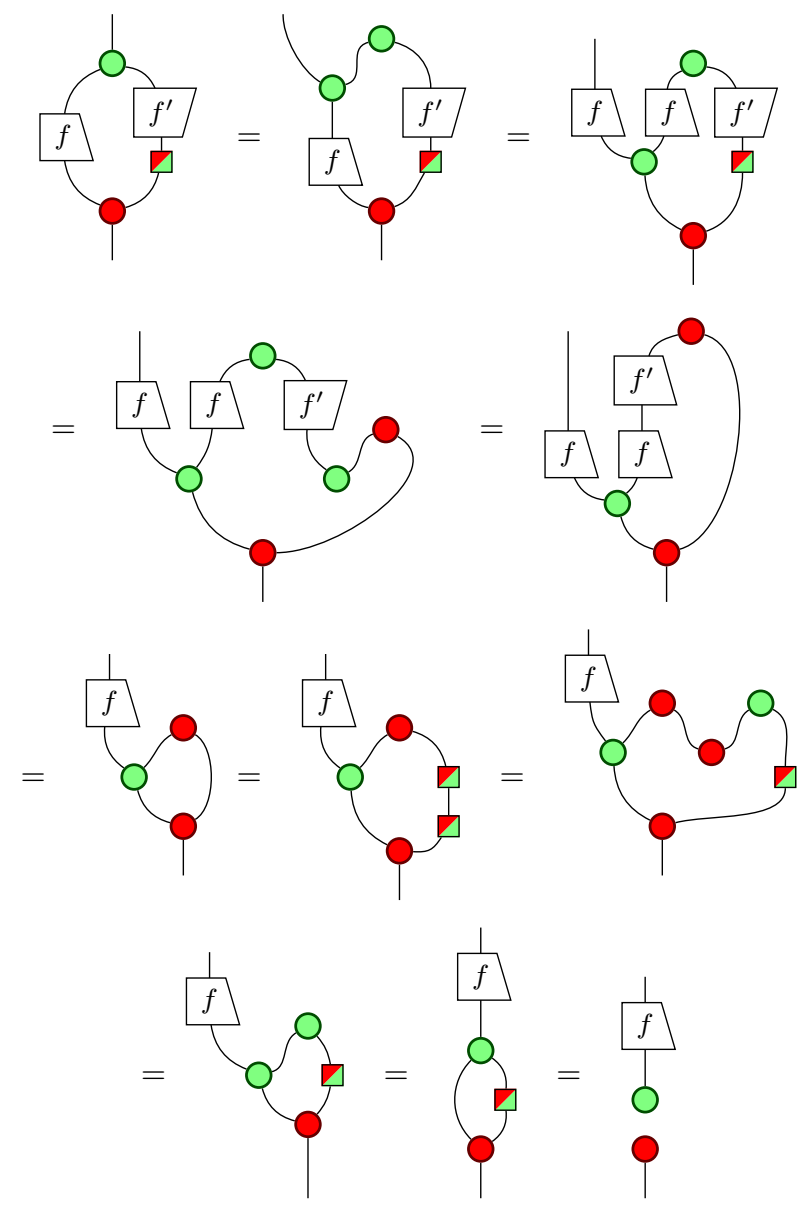

by a similar argument we obtain

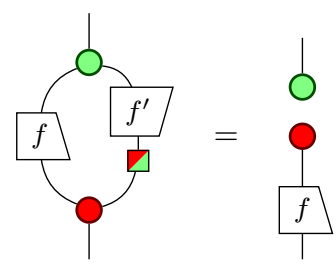

It is easy to see the following

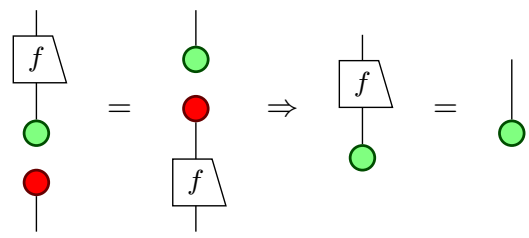

and hence, $\left(-f^{\prime}\right)+f^{\dagger}=0$ as required.

Theorem 6.22. Let $f \in \mathbf{I F}$ be an invertible morphism; if $f \in \mathbf{H A}$ then $f$ is unitary.

Proof. By proposition 6.14, all morphisms in $\mathbf{I F}(1,1)$ are $\bigcirc$-real and $\bigcirc$-real, so in particular $f^{-1}$ is. Since $f \in \mathbf{H A}$ it is a bialgebra morphism. Hence the result follows from Lemma 6.21

Combining the preceding result with Lemma 6.20 shows that the invertible elements of the rings $R$ and $R^{\mathrm{op}}$ must coincide. However, we can do better by appealing to this theorem of Bonchi et al:
Theorem 6.23. [9] Prop. 3.7] Let Mat $R$ denote the category of matrices valued in the ring $R$; then $\mathbf{H A} \simeq \operatorname{Mat} R$ is an isomorphism of PROPS.

Theorem 6.24. Let $f \in \mathbf{I F}$ be invertible; then $f \in \mathbf{H A}$ iff $f \in \mathbf{H A}^{\mathrm{op}}$

Proof. By Theorem 6.22 $f^{-1}=f^{\dagger} \in \boldsymbol{H A}^{\mathrm{op}}$. However by Theorem 6.23 the inverse of an $R$-valued matrix is again an $R$-valued matrix, hence $f^{-1} \in \mathbf{H A}$

Remark 6.25. A priori there are no invertible elements of $R$ other than the identity and the antipode in $\mathbf{I F}(1,1)$. However, when we consider the finite dimensional collapse of IF in Section 8 the this will no longer be true. (By Lemma 6.18 it suffices to consider $\mathbf{I F}(1,1)$.)

\section{Set-like elements and classical maps}

Recall that $\psi: I \rightarrow A$ is called set-like (also called group-like or classical) for the coalgebra $\delta: A \rightarrow A \otimes A$ if $\delta(\psi)=\psi \otimes \psi$. Set-like elements of a $\dagger$-SCFA correspond to the eigenstates of an observable in quantum mechanics, hence they are of great importance for applications. They have many useful properties, which we now explore.

The following is standard; see [35].

Lemma 7.1. Let $(\mu, \delta, \eta, \epsilon)$ be a bialgebra; then:

1. $\eta$ is set-like.

2. If $\psi$ and $\varphi$ are set-like then $\mu(\psi \otimes \varphi)$ is set-like.

3. If this bialgebra is a Hopf algebra with antipode s then $\mu(\psi \otimes$ $(s \circ \psi))=\eta$.

Hence the set-like elements form a monoid for every bialgebra and a group for every Hopf algebra.

In an IF-algebra we have two coalgebras, and hence two ways

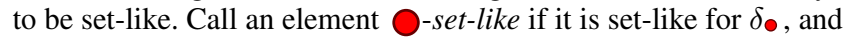
similarly for $\delta_{0}$.

Lemma 7.2. Let $k$ be a $\bigcirc$-set-like element in some IF-algebra; then $k$ is $\bigcirc$-unbiased iff it is O-real.

Proof. If $k$ is $\bigcirc$-real, we have $s \circ k=k_{\bullet}$, hence by Lemma 7.13 we have $\eta_{\bullet}=\mu_{\bullet}(k \otimes(s \circ k))=\mu_{\bullet}\left(k \otimes k_{\bullet}\right)$ which implies $k$ is $\bigcirc$-unbiased by Lemma 4.5 Conversely, if $k$ is $\bigcirc$-unbiased then, by Lemma 7.1 and the uniqueness of inverses, we have $s \circ k=k_{\bullet}$ from which $k^{\top}=k^{\circ}$ follows.

Remark 7.3. Again, this clarifies the "classical points are real" assumption of [16] - in that work $\bigcirc$-set-like elements are separately assumed to be both $\bigcirc$-real and $\bigcirc$-unbiased. In vector space models, $k$ being $\bigcirc$-real means that all its matrix entries are real when written in the orthonormal basis defining $\bigcirc$; this seems a very natural property to demand of the basis vectors themselves! However there is no a priori reason why this should coincide with being $\bigcirc$ -unbiased; it is surprising that these properties are axiomatically equivalent.

Corollary 7.4. Let $\delta_{\bullet}$ and $\delta_{\circ}$ be a pair of interacting $\dagger-S C F A s$ and suppose that the $\mathrm{O}$-set-like elements are also $\mathrm{O}$-real, for $\mathrm{O} \in\{\mathrm{O}, \mathrm{O}\}$; then $\mathrm{O}$-set-like elements form a subgroup of the O-unbiased points and vice versa.

By Lemma 4.5 we know that each $\bigcirc$-unbiased point $\alpha$ determines a $\bigcirc$-phase $\Lambda_{\bullet}(\alpha)$. Phases that are constructed from $\bigcirc$-setlike points are called $\bigcirc$-classical. It follows immediately from Corollary 7.4 that the $\bigcirc$-classical maps form a subgroup of the O-phases. 
Lemma 7.5. No ○-phase can also be $\bigcirc$-classical.

Proof. If $k: I \rightarrow A$ is $\bigcirc$-set-like then $\Lambda_{\circ}(k)=k \circ k^{\circ}$, which is a projector, hence not unitary and therefore not a phase.

Bearing Lemmas 7.2 and 7.5 in mind we wish to consider interacting Frobenius algebras where we have a given subgroup of phases for one colour which are classical for the other.

Definition 7.6. Let $G_{K}$ and $H_{K}$ be subgroups of abelian groups $G$ and $H$ respectively. Define the $\dagger$-PROP $\operatorname{IFK}\left(G \geq G_{K}, H \geq H_{K}\right)$ as the quotient obtained by imposing on $\mathbf{I F}(G, \bar{H})$ the equations

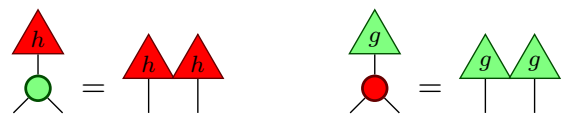

for each $g \in G_{K}$ and each $h \in H_{K}$.

Remark 7.7. Note that in complex Hilbert space models we must have $H_{K} \cong G_{K}$ but there are concrete models in which this is not the case. For example, consider the group algebra of $\mathbb{Z}_{4}$ over the reals. The $\bigcirc$-set-like elements correspond to the elements of $\mathbb{Z}_{4}$ but the $\bigcirc$-set-like elements correspond with the group homomorphisms $\mathbb{Z}_{4} \rightarrow \mathbb{R}^{\times}$, of which there are only two. Hence as groups the set-like elements for the respective colours are not isomorphic.

Lemma 7.8. If $h: 1 \rightarrow 1$ is $\bigcirc$-classical then it commutes with $\delta_{\circ}$ and $\mu_{\circ}$.

In the $\dagger$-PROP $\mathbf{I F}(G, H)$, by definition the only phases for the respective colours are $G$ and $H$. However, the presence of classical maps in $\operatorname{IFK}\left(G \geq G_{K}, H \geq H_{K}\right)$ changes this.

Theorem 7.9. Let $\alpha$ be a $\bigcirc$-phase and $k$ a $\bigcirc$-classical map then $k \circ \alpha \circ k^{\dagger}$ is a $\bigcirc$-phase.

Proof. By its construction $k \circ \alpha \circ k^{\dagger}$ is evidently unitary. We need to show that it is a pre-phase. Consider
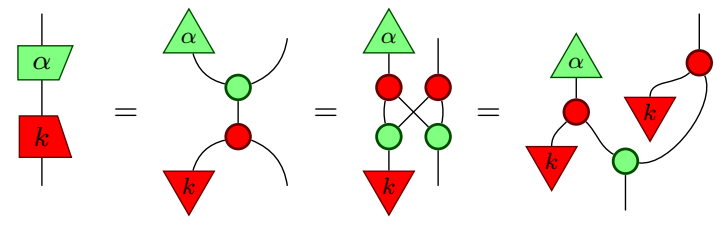

hence we have

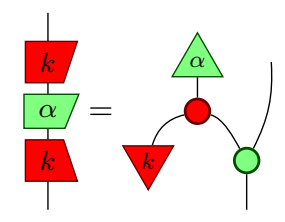

as required.

While Lemma 7.5 tells us that the $\bigcirc$-phases and $\bigcirc$-classical maps are disjoint as groups, there is a degree of interaction between $\bigcirc$-phases and $\bigcirc$-classical maps: the classical maps act on the phase group, to produce new phases.

Lemma 7.10. For $H_{K}$ the group of $\bigcirc$-set-like elements and $\Phi_{\circ}$ the group of $\bigcirc$-phases, there is a group action $\bullet: H_{K} \times \Phi_{\circ} \rightarrow \Phi_{\circ}$.

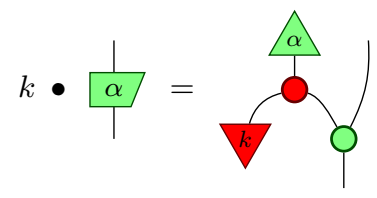

for $\alpha \in \Phi_{\circ}$ and $k \in H_{K}$
Theorem 7.11. The set of morphisms obtained by freely composing $\mathrm{O}$-phases and $\mathrm{O}$-classical maps is a group isomorphic to $\Phi_{\circ} \rtimes_{\varphi} H_{K}$, the (outer) semidirect product of $\Phi_{\circ}$ and $H_{K}$ over the action •.

We end this section with an important lemma which relates the ring structure and the classical maps.

Lemma 7.12. Let $\mathbf{n} \in R$ be an internal integer, and $k: A \rightarrow A$ be a $\bigcirc$-classical map then $\mathbf{n} \circ k=k^{n} \circ \mathbf{n}$.

Proof.

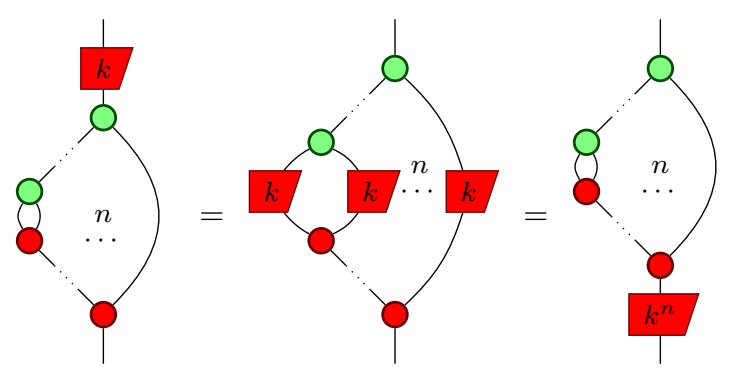

\section{Collapse to finite dimension}

It is well known that if a vector space $A$ supports a Frobenius algebra then $A$ must be finite dimensional. As the last part of our story we incorporate this fact into our axiomatic framework. Recall that in a monoidal category an object $A$ is said to have enough points if, for all morphisms $f, g: A \rightarrow B$, we have

$$
(\forall x: I \rightarrow A, \quad f x=g x) \Rightarrow f=g .
$$

In vector spaces an even stronger form of extensionality is present: two linear maps are equal if they agree on all elements of a basis for the space. Further, we have the following:

Lemma 8.1. Let $\mathbb{k}$ be a field, $A$ a $\mathbb{k}$-vector space, and $\delta: A \rightarrow A \otimes$ A a coalgebra. The set-like elements of $\delta$ are linearly independent.

Proof. See [34 Proposition 7.2].

This motivates the following definition.

Definition 8.2. Let $A$ be an $\operatorname{IFK}\left(G \geq G_{K}, H \geq H_{K}\right)$-algebra; then $A$ has enough $\bigcirc$-set-like elements if

$$
\left(\forall k \in H_{K}: f \circ k \circ \eta_{\bullet}=g \circ k \circ \eta_{\bullet}\right) \Rightarrow f=g
$$

holds for all $f, g: A \rightarrow B$.

By lemma 8.1 it follows that an IFK-algebra in Vect $_{\mathbb{k}}$ has enough $\mathrm{O}$-set-like elements iff the $\mathrm{O}$-set-like elements form a basis. This suffices to determine $\delta_{\circ}$ uniquely, while the group structure of $H_{K}$ determines $\mu_{\bullet}$, and the whole thing is just the group algebra $\mathbb{k}_{k} H_{K}$; cf. Section 3 The dimension of the underlying vector space $A$ is then $\left|H_{K}\right|$.

Since arguments that depend on having enough set-like points are quite common in the categorical quantum mechanics literature, we impose this condition to define the final PROP of this paper. Note, per Remark 7.7 asking for both $G_{K}$ and $H_{K}$ to be enough points is too strong, so we just pick one.

Definition 8.3. Let $\operatorname{IFK}_{\mathbf{d}}\left(G \geq G_{K}, H \geq H_{K}\right)$ denote the $†$ PROP obtained by quotienting $\operatorname{IFK}\left(G \geq \bar{G}_{K}, H \geq H_{K}\right)$ by the equation schema $\left(*\right.$ ) above; this has the effect of ensuring that $\mathbf{I F K}_{\mathbf{d}}$ has enough $\bigcirc$-set-like elements.

Recall that the exponent of a finite group is the least non-zero $n$ such that $g^{n}=1$ for all $g$. 
Theorem 8.4. Suppose $H_{K}$ is finite, and let d be its exponent; then in $\mathbf{I F K} K_{\mathbf{d}}$ the internal integers are the finite ring $\mathbb{Z}_{d}$.

Proof. Applying lemma 7.12 we have

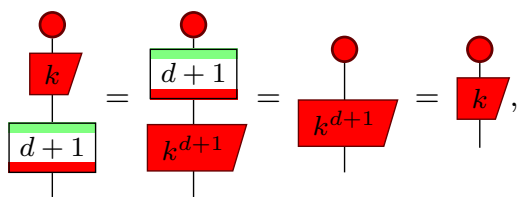

for each $k \in H_{k}$. Since $H_{K}$ is enough points, $\mathbf{d}+\mathbf{1}=\mathrm{id}$, from whence $\mathbf{n}=\mathbf{n}+\mathbf{d}$ for all internal integers $\mathbf{n} \in R$.

The following observation follows directly from Theorem 8.4 and Lemma 6.21

Corollary 8.5. If $n \in \mathbb{Z}_{d}$ has a multiplicative inverse then $\boldsymbol{n} \in \mathbb{Z}$ is unitary. In particular, if the group $H_{K}$ has prime exponent then every non-zero internal integer $\boldsymbol{n} \in R$ is unitary.

Theorem 8.6. Let $d$ be the exponent of $H_{K}$ and $\boldsymbol{n} \in R$ be an internal integer then the following are equivalent:

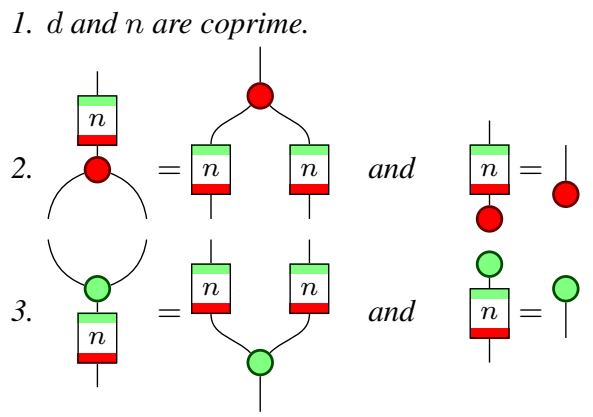

Proof. (1) $\Rightarrow(2)$ and (3): The integers $n$ and $d$ are coprime iff $n$ is invertible in $\mathbb{Z}_{d}$, in which case $\mathbf{n} \in R$ is invertible. By Theorem 6.24 the invertible members of $R$ and $R^{\text {op }}$ coincide. Since $\mathbf{n} \in R^{\mathrm{op}}$ it is a bialgebra morphism for $\mathbf{B}^{\text {op }}$ which implies both (2) and (3).

$(2) \Rightarrow(1)$ : Since $\mathbf{n} \in R$ it commutes with $\left(\mu_{\bullet}, \eta_{\bullet}\right)$, by assumption it commutes with $\left(\delta_{\bullet}, \epsilon_{\bullet}\right)$; hence it is a Frobenius algebra morphism, and by Lemma 4.2 it is invertible in $R$; hence $n$ is coprime to $d$.

(3) $\Rightarrow$ (1) follows by the same argument as above.

In the case where $H_{K}$ has prime exponent, this theorem demonstrates the kind distributive equation between $\mathbf{H A}$ and $\mathbf{H A}{ }^{\mathrm{op}}$ conspicuously absent from Section 6 Indeed equations of this type take part in the distributive law used to construct the PROP $\mathbb{I} \mathbb{H}_{R}^{S p}$ in [9]. However, as we now see, such a law is not possible in our more general setting.

Theorem 8.7. There is no distributive law of PROPs

$$
\tau: \mathbf{F} G ; \mathbf{F} H \rightarrow \mathbf{F} H ; \mathbf{F} G
$$

such that $\boldsymbol{F} H ; \boldsymbol{F} G$ is isomorphic to $\mathbf{I F}(G, H)$.

Proof. If such distributive law exists then for every composable pair

$$
n \stackrel{g_{1}}{\longrightarrow} l \stackrel{h_{1}}{\longrightarrow} m
$$

in $\mathbf{F} G ; \mathbf{F} H$, there must exist an equal composable pair

$$
n \stackrel{h_{2}}{\longrightarrow} k \stackrel{g_{2}}{\longrightarrow} m
$$

in $\mathbf{F} H ; \mathbf{F} G$. Consider the case $n=l=m=1$; then $g_{1}$ and $h_{1}$ are just group elements from $G$ and $H$ respectively. Applying the
Generalised Spider theorem in $\mathbf{F} H$ and $\mathbf{F} G$ separately, we must have

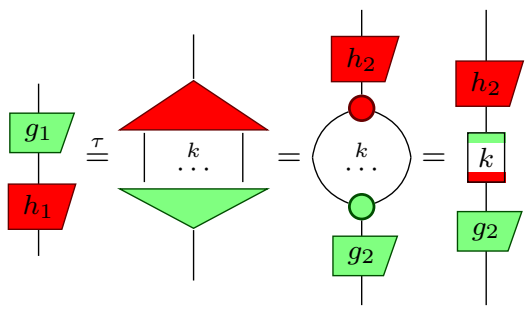

for some $g_{2} \in G$ and $h_{2} \in H$. From this we have

$$
g_{2}^{\dagger} h_{1} g_{1} h_{2}^{\dagger}=\mathbf{k}
$$

The lefthand side is always unitary, but the righthand side is unitary iff $\mathbf{k}$ is invertible in the internal integers $R$; hence by an appropriate choice of model—choose $R=\mathbb{Z}_{k}$ for example—this equation does not hold.

Remark 8.8. Note that even if we restrict to our attention to the case where $R$ is a field, then the phase groups provide an obstruction to a distributive law. For example, consider the standard model of IFK $_{\mathbf{d}}\left(S^{1}, \mathbb{Z}_{2}, S^{1}, \mathbb{Z}_{2}\right)$ over the complex numbers. In this model the unitaries generate the group $\mathrm{SU}(2)$; if the distributive law held it would imply that each $u \in \mathrm{SU}(2)$ could be expressed as two orthogonal rotations, rather than the known three of Euler's decomposition.

\section{Conclusions and future work}

In this paper we have described a sequence of PROPs based on stronger and stronger interactions between a pair of Frobenius algebras augmented with phase groups. At each step another feature of quantum mechanics is introduced, approaching closer to the full theory. Since each PROP is parameterised by the phase groups, we can view them as freely constructed quantum-like theories with the given dynamics. Further, we have shown that, unlike the case of interacting Hopf algebras [9], such theories arise via distributive laws of PROPs only in special circumstances.

Comparison to "Interacting Hopf algebras". The similarities between our system and that of Bonchi, Sobocinksi, and Zanasi [9] are striking. Taking the Frobenius structure as primitive yields almost the same theory as starting with the Hopf structure, and requires fewer axioms to be imposed. The main extra ingredient in IF are the phase groups, which play rather badly with the Hopf algebra structure as Lemma 6.18 shows; we will ignore them and focus on $\mathbf{I F}(1,1)$. Unlike in $\mathbf{I F}$, all the PROPs of [9] have trivial scalars; this forces the generating object to be 1-dimensional.

As noted in Section 6 IF contains both $\mathbf{H A}$ and $\mathbf{H A} \mathbf{A}^{\mathrm{op}}$; however it does not validate any of the axioms concerning the invertibility of the ring elements, nor their commutation with the "wrong" bialgebra map 5 Here Bonchi et al rely on the assumption that $R$ is a PID. However this assumption fails in, e.g., $\mathbb{C Z}_{4}$ which is a perfectly good model of IF. However, in prime dimensional models, Corollary 8.5 implies that these axioms are validated, hence:

Theorem 9.1. Every $\mathbf{I F K}_{\mathbf{d}}$ algebra of prime dimension includes a copy of $\mathbb{I} \mathbb{H}_{\mathbb{Z}}^{S p}$, where $\mathbb{Z}$ denotes the internal integers, and this coincides with the image of $\boldsymbol{I F}(1,1)$, modulo scalar factors.

Comparison to ZX-calculus. The main inspiration for this approach is the ZX-calculus. Writing $S^{1}$ for the circle group, the PROP IFK $_{\mathbf{d}}\left(S^{1}, \mathbb{Z}_{2}, S^{1}, \mathbb{Z}_{2}\right)$, contains all the elements and most of the equations of the ZX-calculus, but there are some key differences.

\footnotetext{
5 to wit: (W1), (W7), (W8), (B1), (B7), (B8), (S1) and (S2).
} 
Firstly, $S^{1}$ is the entire phase group i.e. no new phases are generated by the action of $\mathbb{Z}_{2}$. Secondly, the ZX-calculus incorporates the Hadamard gate, which is a definable map which exchanges the colours. In consequence, the sets of $\mathrm{O}$ - and $\bigcirc$-unbiased points are not disjoint in the ZX-calculus. We will explore necessary and sufficient conditions for such a map to exist abstractly in future work; a connection with Gogioso and Zeng's [22] seems likely.

Further work Many interesting algebraic properties of IF and its relatives remain unexplored: most notable is role of the semidirect product in the phase group (Theorem 7.11, and the possibility to define Hadamard transforms purely abstractly. A tempting next phase of development would to investigate topological features, by considering the case of Lie groups. Finally we note that in the models of the ZX-calculus (although not derivable) we have the Euler decomposition for $\mathrm{SU}(2)$ giving every unitary as a composition of at most three unitaries. An abstract understanding of this would be most valuable.

\section{References}

[1] Samson Abramsky and Bob Coecke. Categorical quantum mechanics. In Handbook of Quantum Logic and Quantum Structures, volume II. Elsevier, 2008, arXiv:0808.1023

[2] Nicolás Andruskiewitsch and Walter Ferrer Santos. The beginnings of the theory of Hopf algebras. Acta Applicandae Mathematicae, 108(1):3-17, 2009, arXiv:0901.2460.

[3] Miriam Backens. The zx-calculus is complete for stabilizer quantum mechanics. New Journal of Physics, 16(9):093021, 2014, arXiv: 1307.7025 .

[4] Miriam Backens. Making the stabilizer zx-calculus complete for scalars. In QPL 2015, 2015, arXiv:1507.03854.

[5] Miriam Backens and Ali Nabi Duman. A complete graphical calculus for Spekkens' toy bit theory. Foundations of Physics, pages 1-34, 2015, arXiv:1411.1618.

[6] John C. Baez and Jason Erbele. Categories in control. arXiv.org, (1405.6881), 2014, arXiv:1405.6881.

[7] David B. Benson. The shuffle bialgebra. In Mathematical Foundations of Programming Language Semantics, volume 298 of LNCS, pages 616-637. Springer Berlin Heidelberg, 1988.

[8] F. Bonchi, P. Sobociński, and F. Zanasi. Interacting bialgebras are Frobenius. In FoSSaCS '14, 2014.

[9] F. Bonchi, P. Sobociński, and F. Zanasi. Interacting Hopf algebras. Technical report, arXiv:1403.7048, 2014.

[10] Filippo Bonchi, Paweł Sobociński, and Fabio Zanasi. A categorical semantics of signal flow graphs. In CONCUR'14, 2014.

[11] Roberto Bruni, Hernán Melgratti, and Ugo Montanari. A connector algebra for P/T nets interactions. In Proc. CONCUR 2011, volume 6901 of $L N C S$, pages 312-326. Springer Berlin Heidelberg, 2011.

[12] Eugenia Cheng. Iterated distributive laws. Mathematical Proceedings of the Cambridge Philosophical Society, 150:459-487, 52011.

[13] B. Coecke and R. Duncan. Interacting quantum observables. In Proc. ICALP 2008, volume 5126 of LNCS, pages 298-310. Springer, 2008.

[14] B. Coecke and E. O. Paquette. POVMs and Naimark's theorem without sums. In Proceedings of the 4th International Workshop on Quantum Programming Languages, volume 210 of Electronic Notes in Theoretical Computer Science, pages 131-152, 2006, arXiv:quant$\mathrm{ph} / 0608072$.

[15] B. Coecke, D. Pavlovic, and J. Vicary. A new description of orthogonal bases. Math. Structures in Comp. Sci., 23(3):555-567, 2013, arxiv:0810.0812.

[16] Bob Coecke and Ross Duncan. Interacting quantum observables: Categorical algebra and diagrammatics. New J. Phys, 13(043016), 2011, arXiv:0906.4725.
[17] Bob Coecke, Ross Duncan, Aleks Kissinger, and Quanlong Wang. Strong complementarity and non-locality in categorical quantum mechanics. In Proceedings of the 27th Annual IEEE Symposium on Logic in Computer Science. (LiCS2012), pages 245-254. IEEE Computer Society Press, 2012, arXiv:1203.4988.

[18] Ross Duncan and Maxime Lucas. Verifying the Steane code with Quantomatic. In Proc.QPL 2013, volume 171 of Electronic Proceedings in Theoretical Computer Science, pages 33-49, 2014, arXiv:1306.4532.

[19] Ross Duncan and Simon Perdrix. Pivoting makes the zx-calculus complete for real stabilizers. In Proc. QPL 2013, volume 171 of Electronic Proceedings in Theoretical Computer Science, pages 50-62. Open Publishing Association, 2014, arXiv:1307.7048.

[20] William Edwards. Non-locality in Categorical Quantum Mechanics. $\mathrm{PhD}$ thesis, Oxford University, 2009.

[21] Bertfried Fauser. Some graphical aspects of Frobenius algebras. In Quantum Physics and Linguistics: A Compositional, Diagrammatic Discourse. Oxford, 2013, arXiv:1202.6380.

[22] Stefano Gogioso and William Zeng. Fourier transforms from strongly complementary observables. Sumbitted to Applied Categorical Structures, 2015, arXiv:1501.04995.

[23] G.M. Kelly and M.L. Laplaza. Coherence for compact closed categories. Journal of Pure and Applied Algebra, 19:193-213, 1980.

[24] J. Kock. Frobenius Algebras and 2-D Topological Quantum Field Theories. Cambridge University Press, 2003.

[25] Stephen Lack. Composing PROPs. Theory and Applications of Categories, 13(9):147-163, 2004, http://www.tac.mta.ca/tac/volumes/13/9/1309abs.html.

[26] S. Mimram. The structure of first-order causality. In Logic In Computer Science, 2009. LICS '09. 24th Annual IEEE Symposium on, pages 212 221, Aug 2009.

[27] Simon Perdrix and Quanlong Wang. The ZX calculus is incomplete for Clifford+T quantum mechanics. arXiv.org, 2015, arXiv:1506.03055.

[28] R. Rosebrugh, N. Sabadini, and R.F.C. Walters. Generic commutative separable algebras and cospans of graphs. Theory and Applications of Categories (Special Issue for CT2004), 15(6):164-177, 2005.

[29] Mehrnoosh Sadrzadeh, Stephen Clark, and Bob Coecke. The Frobenius anatomy of word meanings i: subject and object relative pronouns. Journal of Logic and Computation, 23(6):1293-1317, 2013.

[30] Mehrnoosh Sadrzadeh, Stephen Clark, and Bob Coecke. The Frobenius anatomy of word meanings ii: possessive relative pronouns. Journal of Logic and Computation, 2014.

[31] P. Selinger. Autonomous categories in which $A \cong A^{*}$. In Proceedings of 7th Workshop on Quantum Physics and Logic (QPL 2010), 2010.

[32] Peter Selinger. A survey of graphical languages for monoidal categories. In New structures for physics, volume 813 of Lecture Notes in Physics, pages 289-355. Springer, 2011, arXiv:0908.3347.

[33] Paweł Sobociński. Nets, relations and linking diagrams. In Algebra and Coalgebra in Computer Science, volume 8089 of LNCS, pages 282-298. Springer Berlin Heidelberg, 2013.

[34] R. Street. Quantum Groups: A Path to Current Algebra. Australian Mathematical Society Lecture Series. Cambridge University Press, 2007.

[35] Moss E. Sweedler. Hopf Algebras. W. A. Benjamin Inc., 1969.

[36] James Worthington. A bialgebraic approach to automata and formal language theory. In Logical Foundations of Computer Science, volume 5407 of LNCS, pages 451-467. Springer Berlin Heidelberg, 2009, arXiv:0807.4553. 